\title{
Desgaste psíquico y vulnerabilidad social de mujeres zapotlenses. Un estudio descriptivo
}

\author{
Psychic wear and social vulnerability of Zapotlenses women: A descriptive study
}

Claudia Saldaña-Orozco ${ }^{* *}$ orcid.org/0000-0003-3268-7209

María de los Ángeles Carrión-García² orcid.org/0000-0002-2818-0301

Ana María Gutiérrez-Strauss ${ }^{3}$ orcid.org/0000-0003-0970-9596

Mónica Alejandra Hernández-Flores' orcid.org/0000-0003-0140-9142

1 Centro Universitario del Sur, Universidad de Guadalajara. Guadalajara, México

2 Asociación de Expertos en Psicosociología Aplicada AEPA, Universitat Oberta de Catalunya (UOC). Catalunya, España

3 Universidad El Bosque. Bogotá, Colombia mujeres zapotlenses. Un estudio descriptivo. Univ. Salud. 2017;19(3):321-329. DoI: http://dx.doi.org/10.22267/rus.171903.94

\section{Resumen}

Introducción: Numerosos estudios han documentado la incidencia de los riesgos psicosociales, y particularmente, del desgaste psíquico en la salud de los trabajadores. No obstante, las mujeres en condición de vulnerabilidad social no han sido objeto de exploración. Objetivo: Caracterizar el desgaste psíquico de una muestra de mujeres vulnerables. Materiales y métodos: Estudio exploratorio, descriptivo, correlacional. Se aplicó la dimensión número 4 de la Batería para el estudio de las condiciones de trabajo de carácter psicosocial (CTCPS-MAC), que evalúa desgaste psíquico y 3 factores particulares (respuesta cognitiva-emocional; respuesta conductual y respuesta fisiológica), a 99 mujeres vulnerables de Zapotlán el Grande. Resultados: Un 75,8 \% de las participantes presentaron condiciones óptimas con calificaciones entre bueno y muy bueno, el $24,2 \%$ entre normal y nocivo, lo cual indica que requieren ayuda inmediata. El factor más afectado fue el número 1, respuesta cognitivo-emocional, el $52,5 \%$ presentó sintomatología negativa. No se encontraron asociaciones significativas entre las variables sociodemográficas estudiadas y el desgaste psíquico. Conclusiones: Los resultados permiten abrir puertas para investigaciones más profundas con la población estudiada con el fin de garantizar salud mental, y calidad de vida en las mujeres en estado de vulnerabilidad social de Zapotlán el Grande.

Palabras clave: Vulnerabilidad social; mujeres; salud mental. (Fuente: DeCS, Bireme).

\begin{abstract}
Introduction: Numerous studies have documented the impact of psychosocial risks, and particularly the psychic wear on workers' health. However, women in a situation of social vulnerability have not been the subject of exploration. Objective: To characterize the psychic wear in a sample of vulnerable women. Materials and methods: An exploratory, descriptive and correlational study was conducted. The dimension number 4 from the Battery for the study of the working conditions of psychosocial character was applied (CTCPS-MAC), which evaluates psychological wear and 3 particular factors (cognitive-emotional response; behavioral response and physiological response) at 99 Vulnerable women of Zapotlán el Grande. Results: A 75.8 \% of the participants presented optimal conditions with ratings between good and very good, $24.2 \%$ between normal and harmful, which indicates that they require immediate assistance. The most affected factor was number 1 (cognitive-emotional response), 52,5\% presented negative symptomatology. There were no significant associations between the sociodemographic
\end{abstract}


variables studied and the psychic wear. Conclusions: The results allow to open doors for deeper investigations with the population studied in order to guarantee mental health and quality of life for women with social vulnerability in Zapotlán el Grande.

Keywords: Social vulnerability; women; mental health. (Source: DeCS, Bireme).

\section{Introducción}

La preocupación por los riesgos laborales se ha centrado históricamente en los riesgos físicos y ambientales, pero últimamente ha crecido la atención a los riesgos psicosociales. En la actualidad, debido a diversos factores, los riesgos psicosociales se han incrementado e intensificado. Según Moreno(1), los datos actuales muestran que sus efectos sobre la salud son amplios e importantes. Una atención integral a la salud laboral requiere atender especialmente los riesgos psicosociales. En este contexto resulta necesario realizar acercamientos teóricos y empíricos a los mismos con el fin de salvaguardar la salud en los trabajadores.

Los riesgos o "factores de riesgo" psicosociales se han definido por la Organización Internacional del Trabajo (OIT) y por la Organización Mundial de la Salud (OMS) como "las interacciones entre el contenido, la organización y la gestión del trabajo y las condiciones ambientales, por un lado, $\mathrm{y}$ las funciones $\mathrm{y}$ necesidades de los trabajadores, por otro"(2). "Estas interacciones pueden potenciar o afectar tanto al bienestar, calidad de vida o a la salud (específicamente psicológica o mental) del trabajador como al desarrollo del trabajo"(3).

Las condiciones de trabajo nocivas pueden tener múltiples repercusiones en la salud de los trabajadores, Rotenberg(4) señala entre ellas la fatiga, los accidentes y las lesiones, los trastornos musculo-esqueléticos, problemas psicológicos, estilos de vida poco saludables, así como el aumento del riesgo de la hipertensión y enfermedades cardiovasculares.

Puesto que los factores psicosociales pueden significar un riesgo latente para la salud, tanto física como mental, y su desatención tiene consecuencias apreciables en los niveles de satisfacción y el bienestar psicológico del individuo, es necesario evaluarlos e identificarlos con el fin de adoptar las medidas que sean necesarias para lograr un entorno laboral favorable, ya que "la prevención psicosocial comienza en el análisis de los factores de riesgo psicosocial"(1).

Internacionalmente, la influencia del contexto del trabajo en la salud de los trabajadores ha venido investigándose en diversos ámbitos laborales(5-7). En particular, en Jalisco, al occidente de México, existen algunos estudios que han evaluado los riesgos psicosociales de trabajadores; uno de ellos fue realizado en el Departamento de Obras Públicas de un ayuntamiento del sur del estado(8) y otro evaluó a trabajadores de 109 microempresas ${ }^{(9)}$. Dichas investigaciones se realizaron con dos instrumentos diferentes creados específicamente para evaluar a población trabajadora, como lo son el ISTAS21 COPSOQ(10) y la Batería para el estudio de la condiciones de trabajo de carácter psicosocial (CTCPS-MAC) ${ }^{(11) .}$

Sin embargo, este interés por estudiar los riegos psicosociales en la población trabajadora no ha alcanzado a otros sectores sociales, en este aspecto, un campo muy poco explorado es el referido a la población que no es esencialmente trabajadora, ya que no existen estudios realizados en esta zona de Jalisco que evalúen los riesgos psicosociales que presenta un sector específico de la población: el de las mujeres vulnerables que son beneficiarias del Sistema para el Desarrollo Integral de la Familia (DIF).

El DIF estatal es un organismo público encargado de la asistencia social del estado de Jalisco(12). En la zona sur, a través del DIF municipal se encarga de apoyar a la población vulnerable mediante distintos recursos y apoyos, uno de ellos son las "despensas con productos básicos y charlas de orientación alimentaria que fomenten los buenos 
hábitos alimenticios, coadyuvando así a mejorar su calidad de nutrición"(12), las cuales se entregan de forma mensual a quien cumpla con los requisitos que indican condiciones de vulnerabilidad.

Para el DIF la vulnerabilidad social no es sólo la insatisfacción de necesidades materiales sino que también reconoce la existencia de conductas discriminatorias y de marginación hacia ciertos

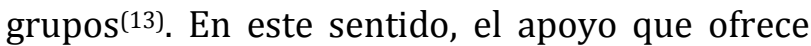
no es sólo para personas con bajos recursos, sino que es prioritario para familias que estén integradas por niños o adolescentes con desnutrición, mujeres embarazadas o en periodo de lactancia, adultos mayores, personas con discapacidad y mujeres maltratadas y/o en abandono(12).

Quizá una razón por la que las investigaciones han dejado de lado el interés por el estudio de los factores psicosociales en este sector de la población es suponer que estas mujeres, en su mayoría, no realizan trabajos fuera del hogar y puesto que, según Murguialday y Bosque $^{(14)}$, socialmente el ocuparse del cuidado de la familia y del hogar no suele verse como un trabajo desde el punto de vista de la salud, sino más bien una prestación de servicios para los que la naturaleza femenina está biológicamente preparada, suele suponerse que realizar labores domésticas no genera ningún tipo de desgaste o daño a la salud.

Sin embargo, dentro del grupo de mujeres vulnerables se encuentran tanto empleadas como amas de casas y dado que diversos estudios indican que las labores domésticas, a pesar de no ser consideradas como un trabajo, sí pueden llegar a representar riesgos para la salud de quienes las desempeñan(14-17), resulta factible evaluar tanto a mujeres que trabajan fuera del hogar como a las que no, con un instrumento diseñado para la evaluación de riesgos psicosociales en población trabajadora, como lo es la Batería para el estudio de las condiciones de trabajo de carácter psicosocial (CTCPS-MAC)(11).
Dicho instrumento evalúa cuatro dimensiones $y$, puesto que cada dimensión se puede valorar por separado, para este estudio se aplicó solamente la dimensión 4, ya que las otras tres se refieren en forma estricta al contexto del área laboral fuera de casa, mientras que los ítems correspondientes a esta dimensión permiten evaluar las alteraciones a la salud ocasionadas por el contexto de trabajo, como el desgaste psíquico.

El término y conceptualización de desgaste psíquico se ha manejado desde distintas posturas, lo cual hace difícil determinarlo teóricamente. Existe una excesiva tendencia a vincularlo al Burnout, el síndrome de quemarse por el trabajo(6), si bien este conlleva un desgaste psíquico, no todo desgaste psíquico es Burnout. Gil-Monte(18) indica que una persona puede sentirse desgastada sin que necesariamente sufra el síndrome de quemarse por el trabajo, por lo que el abordaje de este estudio se sitúa en el desgaste en sí, entendido éste como "una serie de sufrimientos que van desde la fatiga o la depresión, pasando por perturbaciones inespecíficas (insomnio, indigestiones, frigidez, dolores musculares, etc.) y que se producen o agravan en relación con situaciones de tensión psicosocial en el trabajo"(2).

Debido al nulo interés que se ha mostrado en el estudio de los factores psicosociales, en especial del desgaste psíquico, en este sector de la población, resulta imprescindible para el DIF, como principal organismo interesado en el bienestar de la población vulnerable, conocer la situación de las mujeres a las que apoya, en función de establecer estrategias, tanto reparadoras como preventivas, que incidan en su bienestar de forma integral y no sólo a través del apoyo material que se les otorga en forma de despensas. Por lo tanto, el objetivo de esta investigación es caracterizar el desgaste psíquico en este sector de la población a través de cada uno de los tres factores que se evalúan con este instrumento (respuesta cognitiva-emocional; respuesta conductual y respuesta fisiológica). 


\section{Materiales y métodos}

Se realizó un estudio exploratorio, descriptivo, correlacional desde un paradigma cuantitativo, con la finalidad de explicar un fenómeno poco conocido, y buscar las tendencias fundamentales a través de la medición de las variables que el instrumento valida.

\section{Participantes}

La muestra del estudio se integró por 99 mujeres seleccionadas mediante muestreo aleatorio simple. Para determinar el universo de la muestra se empleó los siguientes criterios de selección:

- Exclusión: mujeres que no cumplan con los requisitos de estado de vulnerabilidad que establece el DIF municipal.

- Inclusión: mujeres que cumplen con los requisitos que solicita el DIF municipal para recibir despensas.

Durante el 2016 el DIF municipal expidió el apoyo a un total de 741 mujeres, las cuales componen el universo de la investigación. El tamaño de la muestra aleatoria simple $(n=99)$ se calculó mediante la fórmula para diseños de datos globales con un nivel de confianza de $95 \%$ y un margen de error de 9,2\%.

\section{Instrumento}

Se utilizó la dimensión 4 "Desgaste psíquico" de la Batería CTCPS-MAC, validada para población iberoamericana. La Batería consta de 75 ítems que agrupan cuatro dimensiones y un total de 14 factores. Su propósito es la evaluación de la percepción que tiene la población trabajadora de las condiciones de trabajo de carácter psicosocial en su propio entorno laboral, así como de los factores individuales y los síntomas subjetivos y alteraciones de la salud. Permite evaluar cuatro dimensiones: Contexto de trabajo, Contenido de trabajo, Factores individuales y Desgaste psíquico e incluye catorce factores psicosociales(11). Puesto que este instrumento permite evaluar cada dimensión por separado, sólo se evaluó la Dimensión 4: desgaste psíquico que evalúa los siguientes factores:
-Factor 1 síntomas subjetivos y alteraciones de la salud-estados psicológicos-respuesta cognitivoemocional.

Este factor evalúa la respuesta cognitiva, que es la forma de entender la realidad y desempeñarse en sociedad, la cual está vinculada a la capacidad natural de los seres humanos para adaptarse a su ambiente. En tanto que la respuesta emocional permite establecer su posición respecto al entorno que lo rodea(19).

-Factor 2 síntomas subjetivos y alteraciones de la salud-estados psicológicos, respuesta conductual.

Este factor evalúa la presencia o ausencia de determinados comportamientos estratégicos de afrontamiento ante las situaciones(19).

-Factor 3 síntomas subjetivos y alteraciones de la salud-estados psicológicos-respuesta fisiológica.

Este factor evalúa la reacción que tiene nuestro organismo ante los estímulos externos de estrés o esfuerzo y que modifican su estado natural(19).

El cuestionario cuenta con dos secciones, la primera concierne a variables sociodemográficas y se utiliza principalmente para obtener datos de la persona que contesta y como control para el investigador. La segunda parte, es la dimensión 4 , que consta de 20 reactivos, utiliza escala Likert de 5 niveles: 1, en extremo desfavorable y negativo al reactivo; 2 , desfavorable y negativo al reactivo; 3 , neutral o indeciso; 4, favorable y positivo al reactivo; y 5, en extremo favorable y positivo al reactivo.

La dimensión fue validada al reportar un alfa de Cronbach de 0,884 . Se recuerda que un valor mínimo de coeficiente alfa de Cronbach de 0,7 es aceptable(20).

\section{Procedimiento}

Se realizó la evaluación en 2016, en las instalaciones del DIF municipal. A las participantes se les explicó el objetivo de la investigación y accedieron a responder de forma voluntaria, se les indicó cómo contestar el 
cuestionario y se les aclaró que en los ítems 16 y 18 en los que se lee: 16 . Me gustan las tareas que realizo en mi puesto de trabajo y 18. Mi trabajo no me satisface, las participantes que laboraban fuera del hogar respondieran en función de su puesto de trabajo, mientras que las se desempeñaban como amas de casa, tomaran sus labores domésticas como si fuese su trabajo.

El desgaste psíquico fue interpretado mediante puntaje global y por factores acorde a la explicación detallada en la escala del instrumento. El análisis de los datos obtenidos se elaboró en el programa Statistical Package for the Social Sciences (SPSS) versión 15.0. Se realizó un análisis estadístico descriptivo y para establecer el grado de correlación entre las variables de estudio se utilizó el coeficiente de correlación de Pearson.

\section{Consideraciones éticas}

Para realizar esta investigación se tuvo en cuenta lo dispuesto en la declaración de Helsinki 1975(21) para investigación con personas. A las participantes se les explicó el objetivo de la investigación y accedieron a responder de forma voluntaria, se mantuvieron las consideraciones éticas de confidencialidad y anonimato. Se tomaron todas las precauciones del caso para respetar su vida privada y para reducir al mínimo el impacto del estudio en su integridad física y mental. Se contó con el aval del DIF municipal de Zapotlán el Grande, quien respalda la investigación. Dicha institución cuenta con el consentimiento informado de las participantes para colaborar en el presente estudio.

\section{Resultados}

La primera parte del instrumento aplicado permitió obtener las características sociodemográficas de las participantes, las cuales se pueden observar en la Tabla 1.

Los datos más significativos son que el 31,3\% de las participantes de la muestra tiene una edad entre los 30 y los 40 años, por esto la tendencia se ubica en la etapa de adultez joven y media y el $21,2 \%$ tiene más de 60 años. El 34,3\% tiene dos hijos y el $21,2 \%$, tres. Un 37,4\% reportó no tener familiares a su cargo, y el 26,2\% declaró tener dos familiares a su cargo. En lo que respecta al estado civil, la mayor incidencia fue la de las mujeres casadas con el $54,5 \%$, seguida por las viudas y las solteras, ambas con el 11,2\%.

Tabla 1. Perfil sociodemográfico de las participantes. Zapotlán el Grande, Jalisco, 2017

\begin{tabular}{|c|c|c|}
\hline Variable & $n$ & $\%$ \\
\hline \multicolumn{3}{|l|}{ Edad } \\
\hline$<20$ & 2 & 2,0 \\
\hline$>20$ a 30 & 17 & 17,2 \\
\hline$>30$ a 40 & 31 & 31,3 \\
\hline$>40$ a 50 & 12 & 12,1 \\
\hline$>50$ a 60 & 16 & 16,2 \\
\hline$>60$ & 21 & 21,2 \\
\hline \multicolumn{3}{|l|}{ Número de hijos } \\
\hline 0 & 11 & 11,1 \\
\hline 1 & 5 & 5,1 \\
\hline 2 & 34 & 34,3 \\
\hline 3 & 21 & 21,2 \\
\hline 4 & 11 & 11,1 \\
\hline 5 & 7 & 7,1 \\
\hline$>5$ & 10 & 10 \\
\hline \multicolumn{3}{|l|}{ Familiares a su cargo } \\
\hline 0 & 37 & 37,4 \\
\hline 1 & 12 & 12,1 \\
\hline 2 & 26 & 26,3 \\
\hline 3 & 12 & 12,1 \\
\hline 4 & 8 & 8,1 \\
\hline 5 & 2 & 2,0 \\
\hline$>5$ & 2 & 2,0 \\
\hline \multicolumn{3}{|l|}{ Estado civil } \\
\hline Soltera & 11 & 11,1 \\
\hline Casada & 54 & 54,5 \\
\hline Divorciada & 5 & 5,1 \\
\hline Viuda & 11 & 11,1 \\
\hline Separada & 10 & 10,1 \\
\hline En pareja & 8 & 8,1 \\
\hline \multicolumn{3}{|l|}{ Formación académica } \\
\hline Leer y escribir & 16 & 16,2 \\
\hline Primaria & 30 & 30,3 \\
\hline Secundaria & 34 & 34,3 \\
\hline Bachillerato & 17 & 17,2 \\
\hline Formación profesional & 1 & 1,0 \\
\hline Licenciatura & 1 & 1,0 \\
\hline \multicolumn{3}{|l|}{ Trabaja } \\
\hline Sí & 42 & 42,4 \\
\hline No & 57 & 57,6 \\
\hline Total & 99 & 100 \\
\hline
\end{tabular}

En cuanto a la formación académica, 34,3\% cursó estudios de secundaria, 30,3\% la primaria y sólo dos de ellas cursaron estudios de formación profesional. Por otro lado, en lo que se refiere al trabajo, la mayoría de las participantes, un $57,6 \%$, declararon no trabajar fuera del hogar, y el 42,4\% manifestó sí hacerlo. Las tendencias en esta población muestran que la mayoría son adultas jóvenes con nivel de escolaridad predominantemente de educación básica. 
Respecto al instrumento aplicado, la dimensión número 4 de la batería de evaluación de factores psicosociales (Tabla 2). Un $75,8 \%$ de las participantes presentaron condiciones óptimas con calificaciones entre bueno y muy bueno, el $24,2 \%$ entre normal y nocivo, lo cual indica que requieren ayuda inmediata. En lo concerniente a cada uno de los factores evaluados, se encontró que en el factor 1, síntomas subjetivos $\mathrm{y}$ alteraciones de la salud-estados psicológicosrespuesta cognitivo-emocional, el $47,5 \%$ presenta una calificación entre bueno y muy bueno; sin embargo, un 33\% mostró una calificación regular y hubo 12 casos en nocivo. Factor 2, síntomas subjetivos y alteraciones de la salud- estados psicológicos, respuesta conductual; el $73,7 \%$ presentaron buenas condiciones, mientras que un 5,1\% fue normal, el $17.2 \%$ regular y $4 \%$ como nocivo. Factor 3 , síntomas subjetivos y alteraciones de la saludestados psicológicos-respuesta fisiológica; el resultado es predominantemente bueno y muy bueno con un $79.8 \%$, no obstante un $20.2 \%$ presentan sintomatología.

Tabla 2. Evaluación de desgaste psíquico

\begin{tabular}{|c|c|c|c|c|c|}
\hline \multirow{2}{*}{ Dimensión y Factores } & \multirow{2}{*}{$\begin{array}{c}\text { Muy bueno } \\
n \quad(\%)\end{array}$} & Bueno & Normal & Regular & Nocivo \\
\hline & & $n \quad(\%)$ & $n \quad(\%)$ & (\%) & $n \quad(\%)$ \\
\hline Dimensión 4 Desgaste psíquico & $20(20.2)$ & $55(55.6)$ & $3(3.0)$ & $19(19.2)$ & $2(2.0)$ \\
\hline $\begin{array}{l}\text { 1.- Síntomas subjetivos y alteraciones de la salud -Estados } \\
\text { psicológicos- Respuesta cognitivo-emocional }\end{array}$ & $16(16.2)$ & $31(31.3)$ & $7(7.1)$ & $33(33.3)$ & $12(12.1)$ \\
\hline $\begin{array}{l}\text { 2.- Síntomas subjetivos y alteraciones de la salud -Estados } \\
\text { psicológicos- Respuesta conductual }\end{array}$ & $33(33.3)$ & $40(40.4)$ & $5(5.1)$ & $17(17.2)$ & $4(4.0)$ \\
\hline $\begin{array}{l}\text { 3.- Síntomas subjetivos y alteraciones de la salud -Estados } \\
\text { psicológicos- Respuesta fisiológica }\end{array}$ & $39(39.4)$ & $40(40.4)$ & $2(2.0)$ & $12(12.1)$ & $6(6.1)$ \\
\hline
\end{tabular}

Al buscar correlaciones entre las variables sociodemográficas de las participantes y los resultados, tanto el global de la dimensión 4 como el de cada uno de sus tres factores, a través del coeficiente de Pearson, se encontró que no existían asociaciones significativas entre las variables, pues sólo hubo una correlación positiva débil entre las variables edad y el factor 1 de la dimensión 4, el que corresponde a síntomas subjetivos y alteraciones de la saludestados psicológicos-respuesta cognitivoemocional (Tabla 3).

Tabla 3. Correlación entre variables

\begin{tabular}{|c|c|c|c|c|c|}
\hline $\begin{array}{l}\text { Variables socio- } \\
\text { demográficas }\end{array}$ & $\begin{array}{c}\begin{array}{c}\text { Correlación de } \\
\text { Pearson }\end{array} \\
\end{array}$ & Dimensión 4 & Factor 1 & Factor 2 & Factor 3 \\
\hline \multirow[t]{2}{*}{ Edad } & Correlación de Pearson & 0,153 & $0,233\left(^{*}\right)$ & $-0,009$ & 0,118 \\
\hline & Sig. (bilateral) & 0,130 & 0,021 & 0,926 & 0,245 \\
\hline \multirow[t]{2}{*}{ Número de hijos } & Correlación de Pearson & $-0,031$ & $-0,001$ & 0,016 & $-0,048$ \\
\hline & Sig. (bilateral) & 0,763 & 0,991 & 0,874 & 0,639 \\
\hline \multirow{2}{*}{$\begin{array}{l}\text { Total de familiares a } \\
\text { su cargo }\end{array}$} & Correlación de Pearson & $-0,075$ & $-0,075$ & $-0,093$ & 0,033 \\
\hline & Sig. (bilateral) & 0,461 & 0,459 & 0,358 & 0,745 \\
\hline \multirow[t]{2}{*}{ Estado civil } & Correlación de Pearson & $-0,015$ & 0,029 & 0,039 & 0,052 \\
\hline & Sig. (bilateral) & 0,885 & 0,773 & 0,699 & 0,612 \\
\hline \multirow[t]{2}{*}{ Formación académica } & Correlación de Pearson & $-0,145$ & $-0,164$ & $-0,094$ & $-0,140$ \\
\hline & Sig. (bilateral) & 0,151 & 0,105 & 0,355 & 0,166 \\
\hline \multirow[t]{2}{*}{ Trabaja } & Correlación de Pearson & $-0,013$ & $-0,072$ & 0,042 & $-0,016$ \\
\hline & Sig. (bilateral) & 0,898 & 0,478 & 0,682 & 0,876 \\
\hline
\end{tabular}

* La correlación es significante al nivel 0,05 (bilateral) 


\section{Discusión}

El DIF municipal de Zapotlán el Grande, donde se llevó a cabo este estudio, promovió la evaluación del desgaste psíquico en una población vulnerable con el propósito de emprender acciones de prevención y mejora como una materia pendiente en la actualidad en este sector social al que se le presta poca atención.

Si bien es cierto que los resultados mostraron que la mayoría de la población estudiada presenta condiciones óptimas de salud, al reportar mayormente calificaciones de bueno y muy bueno, tanto en el resultado global de la dimensión 4 como en cada uno de sus tres factores; no se pueden ignorar los resultados en normal, regular y nocivo que indican la necesidad de una intervención.

No se encontró una relación significativa entre las variables sociodemográficas y el desgaste psíquico. Es decir, que las variables: edad, número de hijos, número de familiares a su cargo, estado civil, formación académica no influyen significativamente en el nivel de desgaste psíquico que presenta la población estudiada.

No fue significativo el hecho de que el $43.4 \%$ de las participantes trabajan fuera de su hogar. En este sentido resulta interesante observar que al comparar lo obtenido en otro estudio realizado con el mismo instrumento pero aplicado sólo a población trabajadora de esta zona ${ }^{(8)}$, se encontraron resultados similares. En dicho estudio, en lo que respecta a la dimensión 4, desgaste psíquico, el 15,1\% de los participantes obtuvo un resultado regular, mientras que el $84,9 \%$ entre bueno y muy bueno. En lo conciernente a cada uno de los factores evaluados, en el primero, que evalúa la respuesta cognitivo-emocional el 77,3\% mostró resultados en bueno y muy bueno, con el $22,7 \%$ con respuestas entre normal y regular. En el factor que evalúa la respuesta conductual, sólo un 7.6\% presentó resultados entre normal y regular, en contraste con el $93.6 \%$ que se encontró en óptimas condiciones. En cuanto al factor número 3 , el de síntomas subjetivos y alteraciones de la salud-estados psicológicos-respuesta fisiológica, mostró un $24.5 \%$ en regular y dos casos de nivel nocivo(8).

En general, los resultados en ambos estudios mostraron una tendencia en la mayoría de la población estudiada a presentar calificaciones de bueno y muy bueno en lo relativo a la medición del desgaste psíquico y a sus tres factores. Aunque, en el presente estudio los resultados óptimos fueron levemente menores. Asimismo, en lo relativo a los tres factores evaluados hay algunas diferencias, pues mientras que en este estudio el factor más afectado, con más participantes en niveles entre normal, regular y nocivo, resultó ser el factor número 1 , síntomas subjetivos y alteraciones de la salud-estados psicológicos-respuesta cognitivo-emocional. En la otra investigación, el factor más afectado fue el número 3, el correspondiente a síntomas subjetivos y alteraciones de la salud -estados psicológicos - respuesta fisiológica(8). De la misma manera, los resultados fueron similares a otros estudios realizados con el mismo instrumento, pero aplicados a trabajadores colombianos $(5,6)$; en estos el desgaste psíquico y sus tres factores mostraron una tendencia a bueno y muy bueno.

Puesto que no se encontraron diferencias significativas entre el desgaste psíquico que presentan las mujeres que obtienen una remuneración por trabajar fuera de su hogar y las que sólo se desempeñan como amas de casa, se difiere de los hallazgos obtenidos en diversos estudios en los que esa variable ha reportado diferencias en la salud de las participantes, mostrando una tendencia a presentar mayor salud en las mujeres que reciben una remuneración por trabajar fuera de su hogar. En una muestra de mujeres radicadas en Toluca, México, Garay y Farfán(22) reportaron mayor depresión en las mujeres que tienen trabajo no remunerado. Por su parte, Ferrer et al.(15), estudiaron a un grupo de mujeres de la Comunidad Autónoma de las Islas Baleares, encontraron que las amas de casa presentaban unos niveles de salud inferiores, tanto en lo que se refiere a sintomatología aguda, como a enfermedades crónicas y a valoración del nivel 
de salud. En España, Escalera y Herránz ${ }^{(23)}$ reportaron que existía una gran diferencia en las variables que explicaban la salud de las mujeres según trabajasen o no fuera de casa y una tendencia hacia una mejor salud por parte de las que tenían un trabajo remunerado. En mujeres brasileñas, la investigación de Senicato et al.(24), encontró que las amas de casa mostraron una calidad de vida relacionada con la salud inferior que las trabajadoras remuneradas.

Es importante realizar otros estudios que permitan profundizar más en este campo tan poco explorado. Se requiere indagar en futuras investigaciones cuáles son las características de las mujeres vulnerables que presenten relaciones estadísticamente significativas con el degaste psíquico, con el fin de posibilitar el diseño de estrategias acordes a las necesidades de este sector de la población.

\section{Conclusiones}

De la muestra de mujeres vulnerables estudiadas, un amplio porcentaje no manifiesta presencia de desgaste psíquico. No obstante, es necesario tomar en cuenta a la población que sí presenta afectación para salvaguardar su salud física y mental. Asimismo, se requiere aplicar una estrategia de intervención preventiva para conservar los resultados óptimos que muestra la mayoría.

El factor 1 que corresponde a síntomas subjetivos y alteraciones de la salud-estados psicológicos-respuesta cognitivo-emocional, resultó ser el más afectado, lo cual interpela a aplicar de forma inmediata estrategias psicoeducativas, para evitar que la salud mental de la muestra estudiada se afecte.

Los resultados de investigación permiten abrir puertas para investigaciones más profundas con la población estudiada, con el fin de garantizar salud mental y calidad de vida en las mujeres en estado de vulnerabilidad de Zapotlán el Grande.

Conflicto de intereses. Los autores declaran que no existen conflictos de intereses.

\section{Referencias}

1. Moreno-Jiménez B. Factores y riesgos laborales psicosociales: conceptualización, historia y cambios actuales. Med Segur Trab. 2011;57:4-19.

2. Organización Mundial del Trabajo/Organización Mundial de la Salud. Factores psicosociales en el trabajo: naturaleza, incidencia y prevención. Ginebra: OIT /OMS; 1984. p. 5-78.

3. López F, Carrión MÁ. Perspectiva organizacional de los factores psicosociales. En: Arellano G, editor. Factores psicosociales en el trabajo, un enfoque multidisciplinario. Aguascalientes: Universidad Autónoma de Aguascalientes; 2006. p. 100-125.

4. Rotenberg L. Fórum: horários de trabalho e saúde. Cad Saúde Pública. 2004;20(6):18-36.

5. Carrión-García MÁ, Gutiérrez-Strauss AM, López-Barón F. Condiciones de trabajo psicosociales y desgaste psíquico en trabajadores de diversos sectores empresariales de Centro-Oriente, Suroccidente y Región Caribe en Colombia. Salud Uninorte. 2014;30(3):31-2.

6. Carrión MÁ, López F, Gutiérrez AM. Influencia de factores negativos del contexto de trabajo en desgaste psíquico de trabajadores en Colombia. Hacia Prom Salud. 2015;20(1):111-25.

7. Carrión-García MÁ, López-Barón F, Alpuente-Abelló L. Estudio cualitativo sobre factores psicosociales de riesgo en profesionales de enfermería. Waxapa. 2010;3:27-34.

8. Saldaña-Orozco C, Ramírez-Lira E, Anaya-Velasco A, Baeza-Alcaraz M. Evaluación de los riesgos psicosociales con el instrumento CTCPS-MAC —batería para el estudio de la condiciones de trabajo de carácter psicosocial- en el departamento de obras públicas de un ayuntamiento del Sur de Jalisco (México). Salud Uninorte. 2014;30(3):293-301.

9. Saldaña Orozco C, Anaya Velasco A, Santoyo F. Riesgos psicosociales para la salud laboral de los trabajadores. Evaluar los riesgos psicosociales para la salud laboral de trabajadores en las microempresas de Ciudad Guzmán, Jalisco. México: Publicia; 2013.

10. Moncada S, Llorens C, Kristensen TS. Método ISTAS 21 (CoPsoQ). Manual para la evaluación de riesgos psicosociales en el trabajo ISTAS 21. Barcelona: Paralelo Ediciones; 2004. 1-182 p.

11. Carrión M. CTCPS-MAC: Batería para el estudio de las condiciones de trabajo de carácter psicosocial. Saarbrücken: Editorial Académica Española; 2014.

12. Sistema DIF Jalisco. Ayuda Alimentaria Directa [Internet]. Guadalajara: Sistema DIF Jalisco; 2017. [Citado el 1 de abril de 2017]. Disponible en: http://sistemadif.jalisco.gob.mx/sitio2013/programas /ayuda-alimentaria-directa

13. Dirección Nacional de Alimentación y Desarrollo Comunitario. Índice de vulnerabilidad social. México: Secretaría de Salud; 2015.

14. Murguialday Martínez B, Bosque Pérez A. Riesgos en el trabajo de oficina. España: Instituto Vasco de la mujer; 1994. 128. 
15. Ferrer Pérez VA, Bosh Fiol E, Gili Planas M. Aspectos diferenciales en salud entre las mujeres que trabajan fuera del hogar y las amas de casa. Psicothema. 1998;10(1):53-63.

16. Soria R, Mayen A. Depresión y hábitos de salud en mujeres empleadas domésticas y amas de casa. Rev Electrónica Psicol Iztacala. 2017;20(1):95-114.

17. Gaxiola E. Bienestar psicológico y desgaste profesional [DP] en amas de casa docentes universitarias. J Behav Heal Soc Issues. 2014;6(1):79-88.

18. Gil-Monte P. Burnout syndrome: ¿síndrome de quemarse por el trabajo, desgaste profesional, estrés laboral o enfermedad de Tomás? Rev Psicol del Trab y las Organ. 2003;19(2):181-97.

19. Carrión-García MÁ. Evaluación de factores psicosociales laborales - CTCPS-mac [Internet]. Barcelona: Carrión-García MÁ; 2016 [citado el 1 de abril de 2017]. Disponible en: http://itpfmac.wixsite.com/misitio/singlepost/2016/10/20/Evaluaci\%C3\%B3n-de-factorespsicosociales-laborales---CTCPS-mac

20. Oviedo C, Campo Arias A. Aproximación al uso del coeficiente alfa de Cronbach. Rev Col Psiqui. 2005;XXXIV(4):572-80.

21. Asociación Médica Mundial. Declaración de Helsinki. Principios éticos para las investigaciones médicas en seres humanos. Tokio, Japón; 2008.

22. Garay López J, Farfán García M. Niveles de depresión, autoestima y estrés en mujeres que tienen un trabajo remunerado $\mathrm{y}$ mujeres que tienen un trabajo no remunerado (amas de casa). Psicol Iberoam. 2007;15(2):22-9.

23. Escalera M, Herránz S. Trabajo y salud en la mujer: análisis comparativo de mujeres con trabajo remunerado y amas de casa. Clínica y Salud. 2000;11(3):195-229.

24. Senicato C, Lima M, Barros M. Are there differences between paid women workers and housewives in health-related quality of life?. Cad Saude Publica. 2016;32(8):e00085415. 